\title{
Lassa fever Outbreak in Nigeria: How Prepared Are We?
}

\author{
Adekunle Olatayo Adeoti*1, Onyekachi Ojiaku² and Louise Sigfrid ${ }^{3}$ \\ ${ }^{1}$ Department of Medicine, Ekiti State University Teaching Hospital, Ado Ekiti, Nigeria
}

${ }^{2}$ Department of Medicine, Ekiti State University Teaching Hospital, Ado Ekiti, Nigeria

${ }^{3}$ Clinical Research Fellow and Public Health Specialist, University of Oxford, United Kingdom

Received: May 23, 2018; Published: May 29, 2018

*Corresponding author: Adekunle Olatayo Adeoti, Department of Medicine, Ekiti State University Teaching Hospital, Ado-Ekiti, Nigeria

\begin{abstract}
Despite the discovery of Lassa fever nearly five decades ago, the disease is still endemic in West Africa. The Nigerian Centre for Disease Control's (NCDC) weekly epidemiological report for the first 10 weeks in the year 2018 shows that there were 157 confirmed cases and 56 deaths from Lassa fever outbreak and an upward trend in the case fatality rate in the last three years. This study aims to identify gaps in disease preparedness and propose recommendations in the event of future outbreaks. Some of the notable challenges impeding on preparedness include the annual repeated morbidity and mortality among healthcare workers who cater for infected patients. Furthermore, there is an observed occurrence of epidemics in regions with rocky terrains and environments with rocky terrains. Recommendations for stronger political commitment and the adoption of a one health approach among others were also proposed in order to avert a possible pandemic.
\end{abstract}

\section{Introduction}

Lassa fever is an acute viral haemorrhagic disease endemic in West African countries [1]. The first confirmed cases of the disease were reported in Nigeria back in 1969 and the disease was named after the town where it first occurred in Lassa, Borno State. The estimated sero-prevalence is $21 \%$ [2]. The virus is a single-stranded RNA virus which belongs to the Arenaviridae family with multi mammate rodent being an animal vector which if infected, sheds the virus in their urine and faeces. There have also been reported cases of person-to-person and laboratory transmissions [1].

\section{Epidemiology}

It is estimated that between 300,000 and 500, 000 cases of Lassa fever are reported in West Africa annually [3,4]. And the peak period of the disease commonly between January and June each year with no age, racial or sex predilection [4]. The epidemiological findings presented in this report are strictly from the published data obtained from the NCDC 2018 weekly updates for the first 10 week of the outbreak in 2018. During this period, there were a total of 622 suspected cases with157 confirmed cases and 56 reported deaths (case fatality of $36 \%$ ). In comparison to two previous years, there is an upward trend in the case fatality rates were $13.15 \%$ and $14.46 \%$ respectively [5].

The graphical illustration (Figure 1) indicates that morbidity rates peaked and plateau between weeks 4 and 6 while mortali ty rates decline. Figure 2 shows the map of Nigeria with the states where confirmed cases were reported in the first 10 weeks in the year 2018. This showed contiguous states affected and a good topographical knowledge of the affected states reveals that they have rocky/hilly areas. In addition, food processing with sun drying along the roads and on the rocks in some of the rural areas as shows in Figure 3.

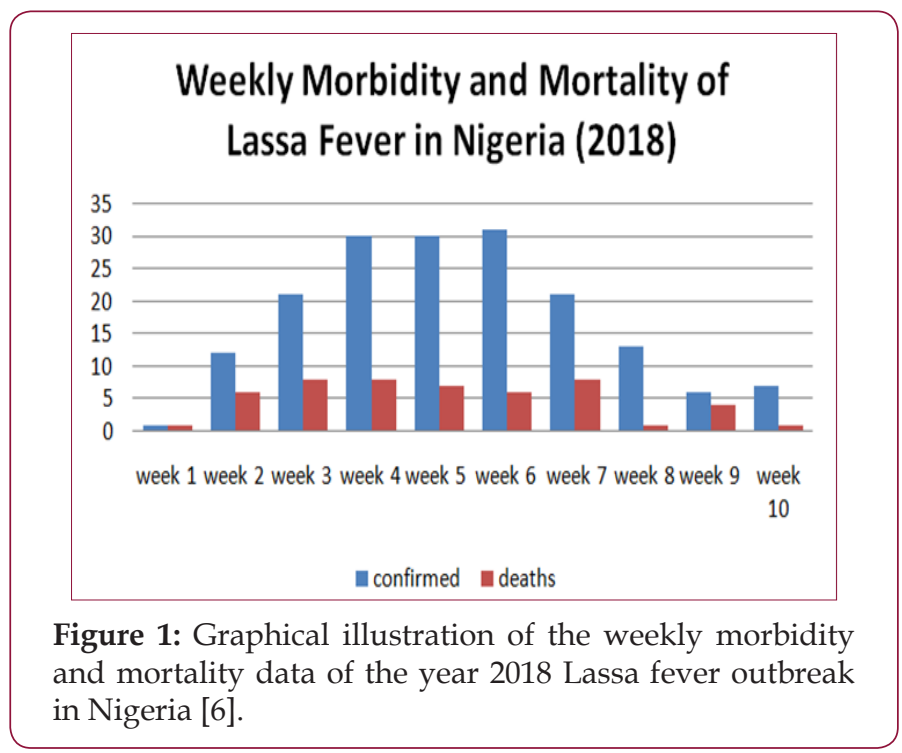




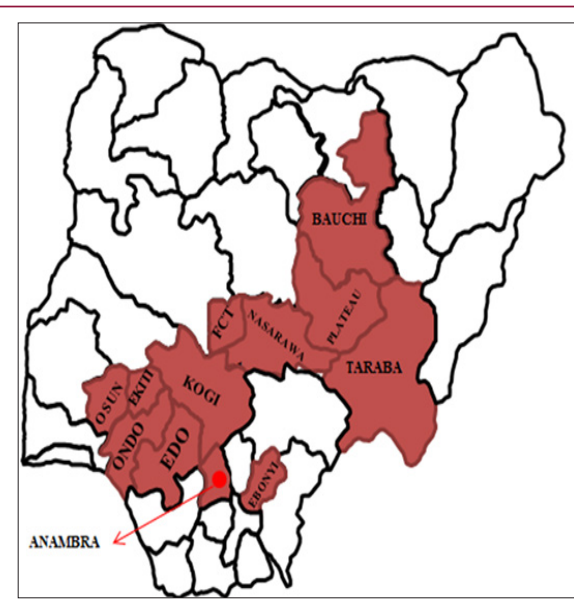

Figure 2: Map of Nigeria depicting States with confirmed cases of Lassa fever in 2018.

\section{Preparedness}

The recurrent annual outbreak of this disease and the increasing death rate among healthcare workers who managed confirmed cases call for a review of the disease preparedness. As evidenced in previous studies, the risk of nosocomial infection is low expect there is direct contact with body fluid of infected patients due to poor infection control measures $[7,8]$. However, personal protective devices (PPE) were reported as inadequate in most healthcare facilities $[9,10]$. Likewise, healthcare workers were reportedly afraid to approach patients in whom the disease was suspected even when PPE was provided [9]. Furthermore, poor knowledge and practices of community members towards the disease, most especially in communities where confirmed cases have been reported [11]. As knowledge regarding its seasonal patterns should not be limited to health workers alone. One of the food processing practices is sun drying of raw food materials by roadsides and flat rocky terrains where they are at risk of being contaminated by excreted waste products the infected vector (Figure 3). Effective communication and coordination by the health ministries is vital to mitigate outbreaks.
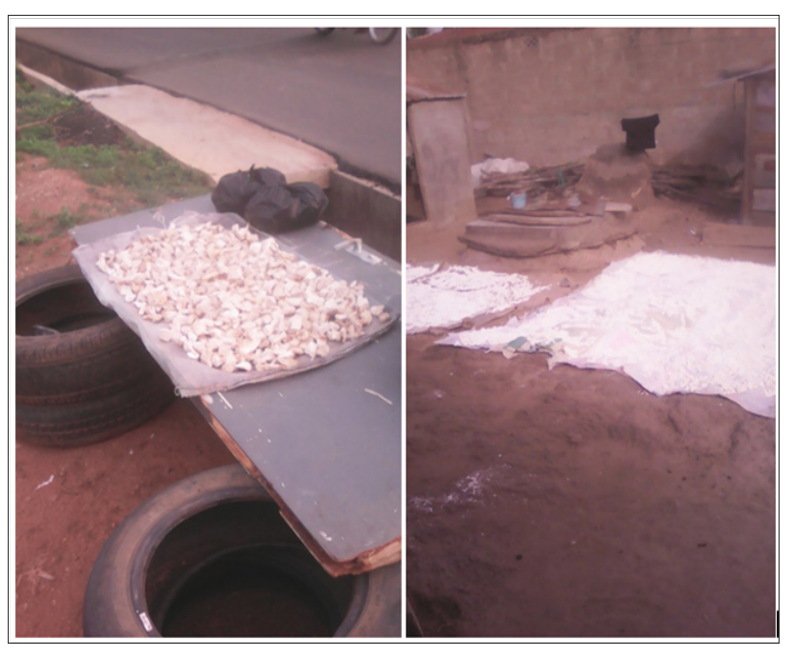

Figure 3: showing sun drying of raw food products by the roadside and plain rocky surface.
In addition, the facilities and healthcare workers are often overwhelmed with the population of suspected cases which have been isolated pending the availability of laboratory confirmation of cases. This is also compounded with the burdens of cases which have been referred to the few facilities where the disease can be diagnosed and managed. A possible consequence of this could be the rejection and those suspected patients, if infected, will further spread the infection in the community. Furthermore, inadequacy of isolation wards could be contributory to the problems faced by centres where the disease is managed. Other challenges involve delayed presentation to a healthcare facility and misdiagnosis due to mimicking other endemic diseases such as malaria which may have been managed primary health centres.

\section{Recommendations}

\section{Standard Case Definition}

There is a need to call for stakeholders meeting to address the annual recurrence and mortality as a result of this disease by the proper dissemination of case definition criteria to all health centres in the country.

\section{Political Commitment}

Policymakers should implement flexible, pragmatic and cost effective strategies to ensure the effective planning and control of the disease during outbreaks. This will include improved funding of the health system with strong legislative support.

\section{Regular Training}

One of the key recommendations is the regular training and retraining of healthcare workers. Apart from refreshing the skill of health workers on prompt identification and management of the disease, this measure will also allay fears in the healthcare professionals and encourage the regular use of PPE which would form a template in the event of an outbreak of any other infectious disease. A standard protocol should be adopted in order to ensure that health workers pay attention to infection control measures while handling suspected cases.

\section{Communication}

Effective communication tailored towards a particular audience is essential during epidemics, especially in areas with the potential to experience the disease outbreak [12]. Furthermore, frequent dissemination of information through the electronic and social media when approaching outbreak periods should be encouraged to help sensitise community members on the characteristics of the disease.

\section{Health Promotion}

Community health workers are also encouraged to be actively involved in the sensitisation of the public in order to encourage good health behaviour as well as eliminate myths and misconceptions surrounding the disease. This will prevent delayed presentation of patients during epidemics.

\section{One Health Approach}

One health is a concept which involves designing and implementation of programmes, policies, legislation and research in 
which various disciplines communicate together in order to realise enhanced public health outcomes [13]. Findings regarding Lassa fever indicate that the disease is rather trans disciplinary, hence, a one health approach which integrates the expertise of the medical, veterinary, agricultural and environmental team is encouraged to help assist where risk factors have been identified in their various disciplines. Moreover, more knowledge gaps would be covered through this multidisciplinary cooperation which could encourage the engagement of the agricultural and bioengineering unit to fabricate ovens which would assist community members in food drying.

\section{Infrastructural Upgrade}

Health infrastructures should be upgraded and redesigned to help accommodate patients especially in epidemic-prone regions. This measure would ensure that hospital departments are not overwhelmed with the influx of cases during an outbreak period as well as the provision of makeshift structures during epidemics for patients comfort.

\section{Establishment of Reference Laboratories}

The establishment of more reference laboratories with close proximity to hospitals would help reduce waiting time to confirm cases as this measure would further prevent the risk of infection among isolated suspected cases. Delayed confirmation of cases could also predispose isolated patients to stigmatisation among community members and this could consequently affect their mental health.

\section{Future Research}

Future research needs to be done to understand the character of the virus. Furthermore, studies need to be carried out to understand the strain of the virus as well as its virulence. The most recent outbreak, epidemiological data indicates that all of the plains where cases were confirmed either has rocky areas or some of its population living on higher plains. It would therefore be of benefit to understand its association with the environment.

\section{Conclusion}

Since the first reported cases of the Lassa fever nearly five decades ago, it is regrettable that it appears that very little has been achieved to prevent mitigate morbidity and mortality from this disease. Available information suggests inadequate preparation towards future outbreaks. This has led to the conclusion that lagging areas could be improved to deliver better quality of services to the patients. A concerted effort towards controlling this is urgently needed, in order to prevent a future pandemic.

\section{References}

1. Centres for Disease Control and Prevention (2015) Lassa Fever.

2. Tomori O, Fabiyi A, Sorungbe A, Smith A, McCormick JB Viral (1988) hemorrhagic fever antibodies in Nigerian populations. The American journal of tropical medicine and hygiene 38(2): 407-410.

3. Ogbu O, Ajuluchukwu E, Uneke CJ (2007) Lassa fever in West African sub-region: an overview. Journal of vector borne diseases 44(1): 1-11.

4. Omeh DJ, Achinge GI, Echekwube PO (2017) Lassa Fever in West Africa: A Clinical and Epidemiological Review 24(6).

5. Nigerian Centre for Disease Control (2017) Preparedness-an important step in outbreak response.

6. Nigerian Centre for Disease Control (2018) Weekly Epidemiological Report. Ebola: Learning From The Past And Preparing For The Future.

7. Ekwere TA, Okafor IP (2013) Hand hygiene knowledge and practices among healthcare providers in a tertiary hospital, south west, Nigeria. International Journal of Infection Control 9(4).

8. Ango UM, Awosan KJ, Adamu H, Salawu S, Sani MM, et al. (2017) Knowledge, Attitude and Practice of Hand Hygiene among Healthcare Providers in Semi-urban Communities of Sokoto State, Nigeria. 26(2): $1-9$.

9. Ajayi NA, Nwigwe CG, Azuogu BN, Onyire BN, Nwonwu EU, et al. (2013) Containing a Lassa fever epidemic in a resource-limited setting: outbreak description and lessons learned from Abakaliki, Nigeria (January-March 2012). International Journal of Infectious Diseases. 17(11): e1011-6.

10. Olalekan AW (2016) Pre-epidemic preparedness and the control of Lassa fever in Southern Nigeria. Research Journal of Health Sciences 4(3): 243-254.

11. Asogun D, Okokhere P, Okogbenin S, Akpede G, Gunther S, Happi C (2010 ) Lassa fever awareness and practices in a Nigerian rural community. International Journal of Infectious Diseases 14: e208.

12. Adeoti AO, Marbus S (2018) The European Respiratory Society course on acute respiratory pandemics: how to plan for and manage them. ERJ open research 4(1): 00156-2017.

13. World Health Organisation (2017) One Health.

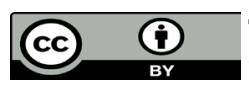

This work is licensed under Creative Commons Attribution 4.0 License

Submission Link: https://biomedres.us/submit-manuscript.php

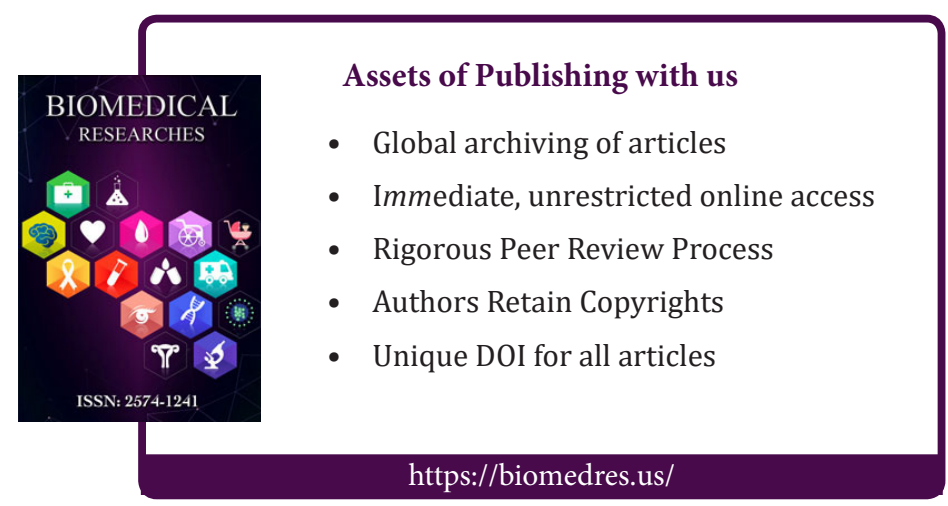

